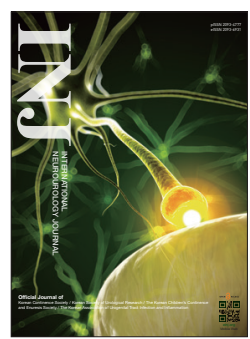

\title{
New Frontiers or the Treatment of Interstitial Cystitis/Bladder Pain Syndrome - Focused on Stem Cells, Platelet-Rich Plasma, and Low-Energy Shock Wave
}

\author{
Chih-Chieh Lin ${ }^{1,2}$, Yun-Ching Huang ${ }^{3}$, Wei-Chia Lee ${ }^{4,5}$, Yao-Chi Chuang ${ }^{4,5}$ \\ ${ }^{1}$ Department of Urology, Taipei Veterans General Hospital, Taipei, Taiwan \\ ${ }^{2}$ Department of Urology, School of Medicine, National Yang-Ming University, Taipei, Taiwan \\ ${ }^{3}$ Division of Urology, Department of Surgery, Chia-Yi Chang Gung Memorial Hospital, Chang Gung University College of Medicine, Chiayi, Taiwan \\ ${ }^{4}$ Department of Urology, Kaohsiung Chang Gung Memorial Hospital, Chang Gung University College of Medicine, Kaohsiung, Taiwan \\ ${ }^{5}$ Center for Shock Wave Medicine and Tissue Engineering, Kaohsiung Chang Gung Memorial Hospital, Chang Gung University College of Medicine, \\ Kaohsiung, Taiwan
}

\begin{abstract}
Interstitial cystitis/bladder pain syndrome (IC/BPS), which is characterized by bladder pain and irritative voiding symptoms, is a frustrating disease without effective treatment. The cause is still largely not understood, although urothelium ischemia/hypoxia, apoptosis, denudation, and infiltration of inflammatory cells are common histopathological findings. The current uncertainty regarding the etiology and pathology of IC/BPS has a negative impact on its timely and successful treatment; therefore, the development of new treatment modalities is urgently needed. Herein, we present advances in our knowledge on this topic and review the potential application of regenerative medicine for the treatment of IC/BPS. This article provides information on the basic characteristics and clinical evidence of stem cells, platelet-rich plasma (PRP), and low-energy shock waves (LESWs) based on a literature review with a search strategy for articles related to IC/BPS, stem cells, PRP, and LESW published in MEDLINE and PubMed. Stem cells, PRP, and LESW, which modulate inflammatory processes and promote tissue repair, have been proven to improve bladder regeneration, relieve bladder pain, inhibit bladder inflammation, and increase bladder capacity in some preclinical studies. However, clinical studies are still in their infancy. Based on the mechanisms of action of stem cells, PRP, and LESW documented in many preclinical studies, the potential applications of regenerative medicine for the treatment of IC/BPS is an emerging frontier of interest. However, solid evidence from clinical studies remains to be obtained.
\end{abstract}

Keywords: Interstitial cystitis/bladder pain syndrome; Platelet-rich plasma; Shock wave; Stem cells

- Fund/Grant Support: This study was supported from Kaohsiung Chang Gung Memorial Hospital (CMRPG8H0452, CRRPG8J0161E) and

National Science Council of Taiwan (106-2314-B-182A-122-MY3).

- Conflict of Interest: No potential conflict of interest relevant to this article was reported by all authors.

\section{INTRODUCTION}

\section{Prevalence of IC/BPS}

Interstitial cystitis/bladder pain syndrome (IC/BPS) is defined as persistent or recurrent chronic pelvic pain or suprapubic dis- comfort perceived to originate from the urinary bladder accompanied with other urinary tract symptoms such as urgency or frequency $[1,2]$. Data on the prevalence of IC/BPS vary widely because of ongoing ambiguity in the definition, differences in the populations studied, and inconsistencies in diag-

Corresponding author: Yao-Chi Chuang (iD https://orcid.org/0000-0002-1468-3792 Department of Urology, Kaohsiung Chang Gung Memorial Hospital, Chang Gung University College of Medicine, No. 123 Ta-pei Road, Niaosong District, Kaohsiung City 83301, Taiwan E-mail: chuang82@ms26.hinet.net

Submitted: April 7, 2020 / Accepted after revision: May 29, 2020 
nostic methods. The prevalence of IC/BPS was estimated to be $2.7 \%$ to $6.5 \%$ and $1.9 \%$ to $4.2 \%$ for US women and men, respectively $[3,4]$. Using the O'Leary-Sant definition of IC symptoms and problem index, the estimated prevalence among women in Finland was 300 per 100,000 [5,6]. The prevalence of IC/BPS in women was $0.261 \%$ and $0.129 \%$ in Korea and Taiwan, respectively $[7,8]$.

\section{Diagnosis of IC/BPS}

Since patients present with a wide range of phenotypes and variable symptoms, they may be significantly misdiagnosed, as it is challenging for clinicians to diagnose IC/BPS. Symptom scores, such as the O'Leary-Sant symptom index, may help to assess patients and can serve as outcome measures for clinical studies. Cystoscopy with hydrodistention is recommended [912]. IC/BPS can be diagnosed in symptomatic patients after excluding cancer, infection, and certain other diseases [10]. Nevertheless, some differences exist in the recommended diagnostic evaluations between the European Association of Urology and the American Urological Association clinical guidelines. Cystoscopy is considered to be required as a diagnostic tool to differentiate between the ulcer and non-ulcer types [13].

\section{PATHOPHYSIOLOGY OF IC/BPS (Fig. 1)}

\section{Urothelial Dysfunction}

The urothelium is characterized both by a highly specialized transitional epithelium forming the inner lining of the urinary bladder and an interface with a high-resistance barrier to ions [14]. Its lining, a protective layer of dense glycosaminoglycans, maintains impermeability to various solutes in the urine. Nevertheless, urothelial abnormalities could be noted based on cystoscopic findings of denudation or thinning, and glomerulations with or without Hunner ulcers in IC/BPS patients [15]. Furthermore, the abnormal expression of urothelial barrier proteins such as tight junction protein zonula occludens-1, uroplakin, E-cadherin, and chondroitin sulfate was observed in the bladders of patients with IC/BPS [16-18].

\section{Increased Apoptosis}

Previous studies have demonstrated that increased numbers of apoptotic cells and decreased numbers of proliferative cells were noted in IC bladder urothelium. Upregulation of inflammatory signals, originating from chronic inflammation in the suburothelium, can result in apoptosis in the bladders of pa-

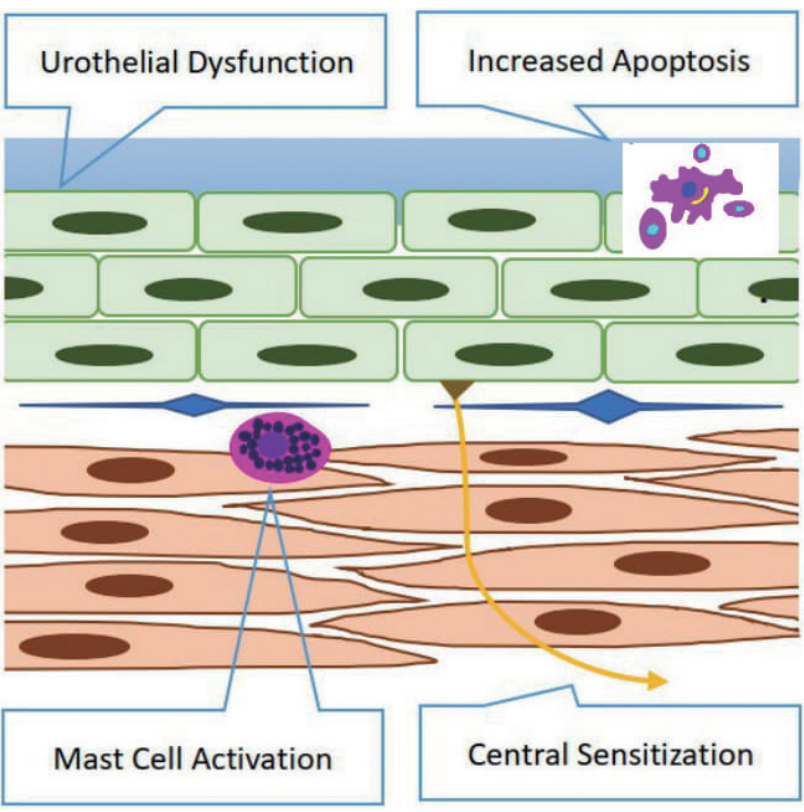

Fig. 1. Pathophysiology of interstitial cystitis/bladder pain syndrome.

tients with IC/ BPS. Shie et al. [19] reported that the apoptotic signaling molecules, such as Bax, cleaved caspase-3, and Bad, were present at higher levels in IC/BPS bladders, and the mechanism of apoptosis was mediated by tumor necrosis factor alpha (TNF- $\alpha$ ) and p38 MAPK [20].

\section{Mast Cell Activation and Neurogenic Inflammation}

In patients with IC/BPS, the bladder has been found to have an increased number of mast cells, which released inflammatory mediators such as histamine and cytokines in response to activation [21], and a more permeable urothelium promoting the influx of potassium ions, with consequent C-afferent fiber activation [22]. The cascade of mast cell activation plays an important role in IC/BPS symptoms, and forms the basis for the use of antihistamines for the treatment of IC/BPS. In addition, the urothelium can release a number of neural signaling molecules, such as adenosine triphosphate and nerve growth factor, which subsequently activate submucosal afferent nerves and mast cells $[22,23]$. These feedback loops result in chronic voiding and pain symptoms, as well as structural changes of the bladder wall in IC/BPS [24].

\section{Central Sensitization}

Central sensitization is a phenomenon of the nervous system that involves enhanced responsiveness of the central neurons, 
even without ongoing nociceptive stimulation from the periphery. Visceral pain may also arise from continuous activation of dorsal horn neurons in the spinal cord, which can induce pain long after inflammation or other problems of the pelvis are cured [25]. This phenomenon explains why some patients with IC/BPS still have significant pain after undergoing cystectomy.

\section{CURRENT TREATMENT OF IC/BPS}

Since the pathogenesis of IC/BPS remains unclear (Fig. 1), the current goals of treatment are largely based on symptomatic relief. All guidelines recommend using patient education to improve patients' understanding of the disease, while also maintaining realistic goals. Oral medical treatment and subsequent intravesical instillation or bladder wall injection follow the failure of first-line management $[13,26]$. The principles of pharmacotherapy for the treatment of IC/BPS are based on (1) controlling the dysfunctional epithelium by continual replenishment of the glycosaminoglycan layer; (2) mast cell deactivation and suppression of allergies; (3) tricyclic antidepressants; (4) anticholinergics or $\beta 3$ agonists; (5) painkillers and nonsteroidal anti-inflammatory drugs; and (6) intravesical botulinum toxin injection and dimethyl sulfoxide instillation.

\section{POTENTIAL NEW THERAPIES}

No optimal treatment has been established as having long-term effectiveness without side effects. Thus, the identification of new therapies that regulate bladder inflammatory processes and reestablish urothelium homeostasis to promote mucosal healing and regeneration may provide new options for IC/BPS patients.

Stem cells, low-energy shock waves (LESWs), and plateletrich plasma (PRP), which modulate inflammatory processes and promote tissue repair, have been proven to relieve bladder pain, inhibit bladder inflammation, and increase bladder capacity in some preclinical studies and clinical studies of IC/BPS.

\section{APPLICATION OF STEM CELL THERAPY IN THE TREATMENT OF IC/BPS}

\section{Mechanism of Stem Cells in the Treatment of IC/BPS}

Stem cells are defined as having the ability to self-renew and to differentiate into lineages of the ectoderm (e.g., epithelium and neuron), mesoderm (e.g., muscle and stroma), and endoderm (e.g., endothelium) [27]. Consequently, the concept of using stem cells for IC/BPS was originally based on the hypothesis that stem cell transplantation into the bladder might replenish the damaged epithelium and neurons [28]. However, more recently, another hypothesis has emerged, according to which transplanted stem cells might provide therapeutic benefits through the paracrine release of anti-inflammatory, pro-angiogenic, antiapoptotic, and/or antioxidative factors [29]. Furthermore, these paracrine bioactive factors are also believed to have the ability to enhance the expression of stem cell trafficking genes, leading to the recruitment of endogenous stem cells to the damaged tissues [30]. In addition, mesenchymal stem cells (MSCs) are known to possess immunomodulatory properties; therefore, they can be transplanted allogeneically or xenogeneically into immunocompetent recipients without the use of immunosuppressants [31]. Due to the lack of reliable tracking molecules/reagents to demonstrate engraftment and/or differentiation of transplanted stem cells, the therapeutic mechanism of stem cells remains controversial. At present, the paracrine effects of transplanted stem cells seem to be more prominent because of their stimulation of the host's own stem cells and adjacent cells $[28,32]$.

\section{Types of Stem Cells for Regenerative Medicine}

Stem cells are classified according to their developmental capacity. Pluripotent stem cells can differentiate into all 3 embryonic germ layers (ectoderm, mesoderm, and endoderm), whereas multipotent stem cells are able to differentiate into specific cell types [33,34]. Although embryonic stem cells (ESCs), which are derived from the inner cell mass of the blastocyst and are an example of pluripotent stem cells, exhibit unlimited differentiation potential both in vitro and in vivo, and could therefore constitute the ultimate source for the generation of various tissues [33], there are significant concerns about their ethical ramifications and their tendency to differentiate into malignant tissues [35]. Another interesting cell type for possible future therapeutic applications is induced pluripotent stem (iPS) cells, which are usually fibroblasts reprogrammed by the introduction of a set of embryonic transcription factors, although teratoma formation has limited their clinical use [36]. Similar to ESCs, iPS cells exhibit high risks of becoming malignant [37]. Therefore, the development of stem cell therapy in the specialty of urology in particular is mostly confined to MSCs, which are multipotent stem cells that are isolated from adult tissues (e.g., bone marrow, umbilical cord blood, adipose tissue, and dental pulp) $[28,32,38]$. 


\section{Preclinical Studies}

To date, there has been little research on the use of stem cell therapy for IC/BPS, and extant research is mostly limited to animal studies. In 2015, Song et al. [39] reported the therapeutic effect of human umbilical cord blood-derived MSCs (UCBMSCs) in a hydrochloric acid ( $\mathrm{HCl}$ )-induced IC/BPS rat model . These investigators injected $1 \times 10^{6}$ human UCB-MSCs into the injured bladder and then assessed the outcomes using conscious cystometry. They found that UCB-MSCs significantly improved epithelial denudation, neurofilament production, and angiogenesis, while decreasing the inflammatory response and mast cell infiltration. Furthermore, they demonstrated that the injected stem cells stimulated urothelial regeneration of the bladder in patients with IC/BPS through the Wnt and epidermal growth factor (EGF) signaling pathways. In 2018, Xie et al. [40] reported similar results after performing intravenous injections of $1 \times 10^{6}$ UCB-MSCs in a cyclophosphamide-induced rat model. They further demonstrated that UCB-MSCs activated the EGF-dependent AKT/mTOR signaling pathway in a cell co-culture model.

In 2016, Hirose et al. [41] presented the healing effects of dental pulp-derived MSCs (DP-MSCs; $2 \times 10^{6}$, injected into the bladder) in a rat model of $\mathrm{HCl}$-induced cystitis. They found that DP-MSCs promoted the healing of damaged bladder tissue through trophic factors, such as fibroblast growth factor 2, vascular endothelial growth factor, monocyte chemoattractant protein-1, growth-related oncogene, and fractalkine.

In 2018, Furuta et al. [42] reported the effects of bladder wall injection of $1 \times 10^{6}$ adipose-derived MSCs (AD-MSCs) in a $\mathrm{HCl}$-induced IC/BPS rat model. They found that bladder capacity and voiding threshold pressure significantly increased in rats treated with AD-MSCs. Furthermore, bladder injection of AD-MSCs significantly decreased nociceptive behavior, mast cell infiltration, and the expression of TNF- $\alpha$, transforming growth factor-beta, and collagen fibers.

Although stem cells have successfully alleviated IC/BPS in rat models, the process of obtaining these stem cells is very complicated. In 2017, Li et al. [43] reported the therapeutic effects of urine-derived MSCs (UD-MSCs), isolated from human voided urine, in a protamine/lipopolysaccharide-induced IC/BPS rat model. They found that an intravenous injection of $1.2 \times 10^{6}$ UD-MSCs restored bladder function by suppressing oxidative stress, inflammatory reactions, and apoptosis. Thus, it appears that UD-MSCs are more advantageous over other stem cell types in that they can be obtained through a simple, noninva- sive, and low-cost approach that does not require a surgical procedure.

As discussed above, several preclinical studies have reported that MSCs are a practical source of cells for treating IC/BPS. However, their limited proliferation during large-scale ex vivo expansion suggests that alternative cell sources are needed to obtain sufficient cell numbers with an appropriate lineage potential to treat patients with IC/BPS. ESCs exhibit unlimited differentiation potential both in vitro and in vivo and can be expanded ex vivo as immortalized cell lines [33]. In 2017, Kim et al. [44] demonstrated that human ESCs can provide almost unlimited multipotent stem cells through spontaneous or controlled differentiation. In a $\mathrm{HCl}$-induced rat model, a single injection of human ESC-derived multipotent stem cells had greater therapeutic efficiency than bone marrow-derived MSCs in reversing urothelium denudation, mast cell infiltration, tissue fibrosis, apoptosis, and visceral hypersensitivity, without any adverse effects. In 2018, Ryu et al. [45] further monitored the behaviors of transplanted human ESC-derived multipotent stem cells in real time using a combination of longitudinal intravital confocal fluorescence imaging and microcystoscopy in a chronic protamine/lipopolysaccharide-induced IC/BPS rat model. They found that the transplanted cells improved voiding function and repaired tissue damage by differentiation into multiple cell types and gradually integrated into a perivascularlike structure.

Although preclinical trials have provided ample data on the utilization of stem cells for regenerative medicine and tissue repair strategies, concerns have been raised that stem cell transplantation may have undesirable consequences, such as malignancy formation. To alleviate such concerns, recent studies have focused on using MSC-conditioned medium, and as reviewed by Adamowicz et al. [46], many growth factors, cytokines, and trophic agents in MSC-conditioned medium can contribute to the amelioration of IC/BPS pathology and create a favorable environment for the regeneration of bladder tissue. Table 1 summarizes the results of preclinical studies.

Although the pathophysiology of IC/BPS remains controversial, hypotheses include urothelial dysfunction, increased apoptosis, mast cell activation, neurogenic inflammation, and central sensitization. However, all of the above preclinical studies used bladder-centric rat models created by intravesical instillation with chemicals to induce the clinical characteristics of IC/ BPS, which are not expected to fully reproduce the various symptoms experienced by human patients. 
Table 1. Summary of preclinical studies on stem cell therapy for cystitis models mimicking IC/BPS

\begin{tabular}{|c|c|c|c|c|c|c|}
\hline First author & $\begin{array}{l}\text { Stem cell } \\
\text { source }\end{array}$ & Stem cell type & $\begin{array}{l}\text { Transplanta- } \\
\text { tion route }\end{array}$ & Tracking of stem cells & Differentiation & Results \\
\hline $\begin{array}{l}\text { Song et al. } \\
\text { [39] }\end{array}$ & Human & UCB-MSCs & $\begin{array}{l}\text { Bladder } \\
\text { injection }\end{array}$ & PKH26 & Yes & $\begin{array}{l}\text { Ameliorated epithelial denudation and abnormal } \\
\text { inflammation, neural networks, and angiogenesis } \\
\text { by activating Wnt signaling pathway. }\end{array}$ \\
\hline $\begin{array}{l}\text { Xie et al. } \\
{[40]}\end{array}$ & Human & UCB-MSCs & $\begin{array}{l}\text { Intravenous } \\
\text { injection }\end{array}$ & No & Unknown & $\begin{array}{l}\text { Alleviated IC/BPS in a chemically induced animal } \\
\text { and cell models by alleviating inflammation, } \\
\text { promoting proliferation, and inhibiting apoptosis } \\
\text { via EGF-dependent AKT/mTOR signaling pathway. }\end{array}$ \\
\hline $\begin{array}{l}\text { Hirose et al. } \\
\text { [41] }\end{array}$ & Human & DP-MSCs & $\begin{array}{l}\text { Bladder } \\
\text { injection }\end{array}$ & $\begin{array}{l}\text { In situ hybridization for } \\
\text { human Y chromosome }\end{array}$ & No & $\begin{array}{l}\text { Promoted healing of chemically induced cystitis } \\
\text { through the following trophic factors: fibroblast } \\
\text { growth factor 2, vascular endothelial growth } \\
\text { factor, monocyte chemoattractant protein-1, } \\
\text { growth-related oncogene and fractalkine. }\end{array}$ \\
\hline $\begin{array}{l}\text { Furuta et al. } \\
\text { [42] }\end{array}$ & Rat & $\begin{array}{l}\text { Adipose-derived } \\
\text { MSCs }\end{array}$ & $\begin{array}{l}\text { Bladder } \\
\text { injection }\end{array}$ & GFP & Unknown & $\begin{array}{l}\text { Decreased the number of mast cells, expression of } \\
\text { TNF- } \alpha \text {, TGF- } \beta \text {, and collagen fibers, and nociceptive } \\
\text { behavior in a chemically induced IC/BPS rat model. }\end{array}$ \\
\hline Li et al. [43] & Human & $\begin{array}{l}\text { Urine-derived } \\
\text { MSCs }\end{array}$ & $\begin{array}{l}\text { Intravenous } \\
\text { injection }\end{array}$ & No & Unknown & $\begin{array}{l}\text { Restored bladder function and histological damage } \\
\text { via decreasing oxidative stress, inflammatory, and } \\
\text { apoptotic biomarkers in a protamine/ } \\
\text { lipopolysaccharide-induced IC/BPS rat model. }\end{array}$ \\
\hline Kim et al. & Human & $\begin{array}{l}\text { ESC-derived } \\
\text { multipotent } \\
\text { stem cells }\end{array}$ & $\begin{array}{l}\text { Bladder } \\
\text { injection }\end{array}$ & GFP & Yes & $\begin{array}{l}\text { Compared with bone marrow-derived MSCs, a single } \\
\text { injection of ESCs-MSCs had superior efficacy in } \\
\text { improving bladder voiding function and } \\
\text { histological damage including urothelium } \\
\text { denudation, mast cell infiltration, tissue fibrosis, } \\
\text { apoptosis, and visceral hypersensitivity. }\end{array}$ \\
\hline $\begin{array}{l}\text { Ryu et al. } \\
\text { [45] }\end{array}$ & Human & $\begin{array}{l}\text { ESC-derived } \\
\text { multipotent } \\
\text { stem cells }\end{array}$ & $\begin{array}{l}\text { Bladder } \\
\text { injection }\end{array}$ & GFP & Yes & $\begin{array}{l}\text { A combination of longitudinal intravital confocal } \\
\text { fluorescence imaging and microcystoscopy in a } \\
\text { chronic IC/BPS animal model demonstrated that } \\
\text { transplanted human ESCs-MSCs improved } \\
\text { voiding function and histological damage by } \\
\text { differentiation into multiple cell types and } \\
\text { gradually integrated into a perivascular-like structure }\end{array}$ \\
\hline
\end{tabular}

IC/BPS, interstitial cystitis/bladder pain syndrome; UCB, umbilical cord blood; MSCs, mesenchymal stem cells; EGF, epidermal growth factor; Akt/mTOR, protein kinase B/mammalian target of rapamycin; DP, dental pulp; GFP, green fluorescent protein; ESCs, embryonic stem cells; TNF, tumor necrosis factor; TGF, transforming growth factor.

\section{Clinical Trials}

Although the potential of stem cell therapy for IC/BPS has been reported in many preclinical models, stem cell therapy is not yet available for clinical use. To date, only 1 clinical trial has assessed the efficacy of stem cells for IC/BPS. In 2019, Lander et al. [47] investigated the effect of combined intravenous and local injections of autologous stromal vascular fraction (SVF) into the pelvic floor in 91 women and 18 men with IC/BPS. Although that study demonstrated that SVF, as an autologous personalized regenerative strategy, showed good safety and efficacy and may have the potential to alleviate IC/BPS, the major limitations were the lack of a control group, inconsistent transplantation cell number, inconsistent cell injection routes in male patients, lack of information on the molecular mechanisms of SVF therapy, and the absence of long-term data. Larger, multicenter, longterm, randomized clinical trials are warranted to elucidate the efficiency of SVF in patients with IC/BPS.

\section{APPLICATION OF PLATELET-RICH PLASMA IN THE TRATMENT OF IC/BPS}

\section{What is PRP?}

PRP is a biological product defined as the portion of the plasma fraction of autologous blood with a platelet concentration above the baseline blood plasma [48]. Generally, the preparation of PRP involves obtaining autologous whole blood from the pa- 
tient, followed by centrifugation and the use of different density gradients to separate plasma from red blood cells and leukocytes. There are several commercial devices available that simplify the preparation of PRP. According to the manufacturers, PRP devices usually achieve a concentration of PRP 2-5 times the baseline concentration. As such, PRP contains not only a high level of platelets, but also the full complement of clotting factors. PRP is rich in a range of growth factors, chemokines, cytokines, and other proteins. The therapeutic effects of PRP on different pathologies may depend on the presence or absence of leukocytes, the activation status of platelets, fibrin architecture, and platelet concentration. Although one might think that a higher platelet count with a higher number of growth factors would lead to better results, this has not been determined yet. The DEPA (dose, efficiency, purity, activation) classification proposed by Magalon et al. [49] may help clinicians to select a PRP product that fits their specific needs for a given indication.

\section{Mechanism of Action of PRP in IC/BPS}

PRP contains numerous growth and cytokines that potentially offer an alternative treatment modality to assist in the healing of bladder mucosa injuries [50]. Platelets develop from bone marrow and contain precursors that can divide and mature. The main function of platelets is to contribute to homeostasis through 3 processes: adhesion, activation, and aggregation [48]. When a vascular lesion is present, platelets are activated, and their granules release factors that promote coagulation. PRP is a natural source of signaling molecules, and upon activation of the platelets in PRP, the P-granules are degranulated, releasing growth factors and cytokines that modify the pericellular microenvironment. Some of the most important growth factors released by platelets in PRP are vascular endothelial growth factor, fibroblast growth factor, EGF, hepatocyte growth factor, insulin-like factor 1 and 2, matrix metalloproteinase (MMP) 2 and 9, and interleukin (IL)-8 [48,50,51]. PRP promotes 4 main actions (proliferation, migration, cell differentiation, and angiogenesis) in the local environment where it is administered.

The precise mechanism by which PRP promotes bladder healing in IC/BPS is not fully understood. IC may result from urothelial barrier dysfunction, which initiates a cascade of neurogenic inflammation [50]. As a result, chronic inflammation and bladder fibrosis would elicit bladder pain and a small bladder capacity. PRP injections into the bladder might provide several growth factors that promote cell proliferation, regeneration, and differentiation to heal the urothelium. PRP may also activate additional inflammatory signaling by its cytokines and leukocytes, switching to an anti-inflammatory phenotype and releasing anti-inflammatory factors. Thus, PRP injections into the bladder might enhance tissue angiogenesis and eliminate refractory neuropathic pain (Fig. 2).

\section{Therapeutic Effects of PRP on IC/BPS}

It has been demonstrated that rabbits' bladders, insulted by $\mathrm{HCl}$ bladder instillation or intraperitoneal cyclophosphamide injection, could be protected by subsequent instillation of PRP into the bladder [52]. PRP treatment can initiate cell proliferation above the basement membrane and protect the urothelium by 48 hours after instillation.

In a pilot clinical study, Jhang et al. [53] performed intravesical injections of PRP 4 times in 1 month $(12 \mathrm{~mL}$ extracted from $50 \mathrm{~mL}$ of whole blood) in 15 refractory IC/BPS patients and found that PRP improved IC symptoms at 1 month of follow-up . No obvious adverse effects were observed in this preliminary study. In addition, the significant posttreatment increases of urinary IL-2 and IL-8 in these IC patients suggest that immune modulation of PRP may play a role in the relief of IC/BPS symptoms. In a subsequent clinical trial, Jhang et al. [54] further reported the outcomes of IC patients who received repeated PRP (10 mL extracted from $50 \mathrm{~mL}$ of whole blood) intravesical injections 4 times in 1 month. In that study, $67.5 \%$ of IC participants showed improvement of IC/BPS symptoms and increased functional capacity at a 3-month follow-up. Hence, the intravesical injection of autologous RPR is promising for treating IC/BPS. The major disadvantage is the need for anesthesia during PRP injection. In summary, intravesical injections of PRP could be a potential treatment for IC/BPS patients, in terms of wound healing, tissue regeneration, and immune modulation. However, there is no consensus regarding PRP preparation or the precise mechanism underlying its effects. Currently, the therapeutic use of PRP in IC/BPS patients is still experimental. Further largescale randomized clinical trials are needed to verify the effects of PRP and its mechanisms of action in IC/PBS.

\section{APPLICATION OF LOW-ENERGY SHOCK WAVES IN THE TREATMENT OF IC/BPS}

\section{Characteristics of Shock Waves}

Shock waves (SWs) are acoustic waves that carry energy from an area of positive pressure to an area of negative pressure, which requires a medium (either water or a coupling gel) for 

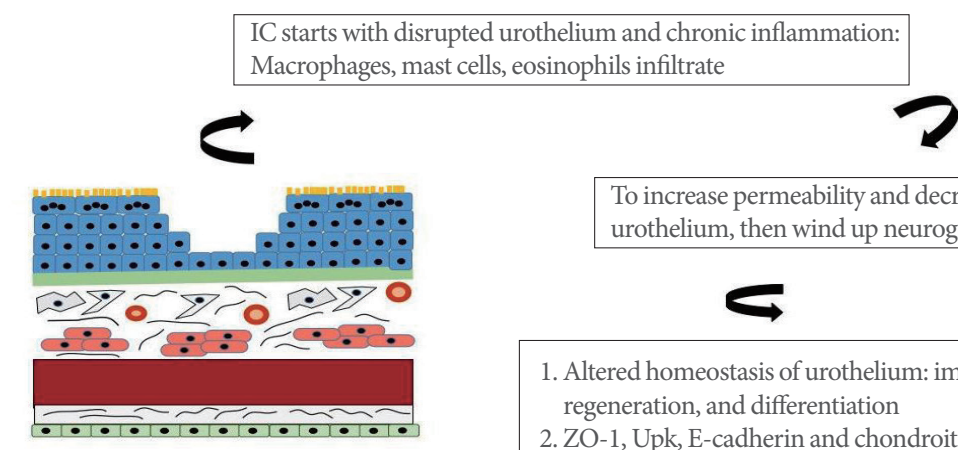

urothelium, then wind up neurogenic inflammation

Treating with PRP, which contains of PDGF, EGF, VEGF, and TGF- $\beta$

\section{$<$}

1. Altered homeostasis of urothelium: imparired proliferation, regeneration, and differentiation

2. ZO-1, Upk, E-cadherin and chondroitin decreased

3. Increased apoptosis (Bax, cleaved caspase-3, and Bad)

4. Pro-inflammatory factor ( $p 38 \mathrm{MAPK}$ and TNFa) activation

5. Lower IL-8

6. Flare up purinergic receptor $\mathrm{P} 2 \mathrm{X} 3$ and nociceptive receptors

7. ATP released

8. Injured cell layers and mature apical umbrella cells in urothelium

1. Promote cell proliferation regeneration and differentiation

2. Secrete several kinds of cytokines and recruited macrophges and

neutrophils, then switching to an anti-inflammatory phenotype and

relese anti-inflammatory factors

3. Promote tissue angiogenesis

4. Promote neuron regeneration

5. Eliminate the refractory neuropathic pain

Fig. 2. Therapeutic potential of intravesical injections of platelet-rich plasma (PRP) in treating interstitial cystitis (IC). PDGF, plateletderived growth factor; EGF, epidermal growth factor; VEGF, vascular endothelial growth factor; TGF, transforming growth factor; MAPK, mitogen-activated protein kinase; ATP, adenosine triphosphate.

propagation in humans. The kinetic energy of SW induces a cavitation effect, followed by a mechanical shear force on the target tissue, causing biological effects. The characteristics of SW include a high peak pressure (up to $100 \mathrm{MPa}$ ), a rapid rise $(<10 \mathrm{~ns})$, a short duration $(<10 \mathrm{msec})$, and a broad frequency spectrum (in the range of $16 \mathrm{~Hz}$ to $20 \mathrm{MHz}$ ) [55,56]. The generation of SW induces a micrometer-sized intense burst of bubbles within and outside of cells, and exerts energy toward the target body tissues [57,58]. SW can be used to target tissues at various depths of penetration, levels of energy flux density $(\mathrm{m}) /$ $\mathrm{mm}^{2}$ ), total energy administration, and focal volumes.

SW at high-energy levels induce irreversible alterations to the cell structure and organelles [58-61]; however, SW at low-energy levels induce a series of cell signaling pathways and activate biological processes such as angiogenesis, cell proliferation, inhibition of apoptosis, peripheral nerve regeneration, and antiinflammation through various mechanisms, depending on tissue type and condition. Nevertheless, studies of dose-response relationships between the intensity of SW and their biological effects remain inadequate.

\section{Rationale for LESW for IC/BPS}

Although the etiology and pathogenesis of IC/BPS are still undetermined, there is increasing support for the hypothesis of neurogenic inflammation, according to which activation of bladder afferent nerves provokes bladder pain and hypersensitivity [62]. Increased apoptosis in the urothelium and levels of hypoxia-inducible factor-1 $a$ have been shown to be mediated by inflammation in IC/BPS $[19,63]$. Therefore, the regulation of inflammatory reactions and the regeneration or repair of urothelium defects could be an attractive option for the management of IC.

High-energy extracorporeal SW therapy has been used to disintegrate urolithiases for 30 years [64]. LESW therapy is used clinically to improve tissue regeneration at tendon-bone junctions, to treat ischemic cardiovascular disorders, and to ameliorate erectile dysfunction [65-67]. LESW therapy has been shown to generate some growth factors or cytokines, to recruit or activate progenitor cells (adult stem cells), and to increase levels of endothelial nitric oxide synthase in the wound or injured organ [65]. In addition, LESW treatment has been suggested as a potentially useful tool for down-regulating nuclear factor-kappa B 
(NF- $\mathrm{BB}$ ) and NF- $\mathrm{kB}$-dependent inflammatory genes, leading to the modulation of the inflammatory process [68].

Our previous study has demonstrated that LESW treatment inhibited nerve growth factor, IL-6, and cyclooxygenase- 2 expression, and blocked bladder pain, inflammation, and overactivity in a cyclophosphamide-induced cystitis model in rats [69]. Chen et al. [70] revealed that LESW significantly alleviated bladder damage by ameliorating inflammation (decreased expression of IL-12, MMP-9, TNF-a, NF- $\mathrm{kB}$, and inducible nitric oxide synthase) and oxidative stress (decreased NADPH oxidase 1 and NOX-2 expression) by using different doses of LESW and cyclophosphamide.

In addition, LESW therapy has been shown to augment the expression of proliferating cell nuclear antigen, downregulate production of proinflammatory cytokines, and improve wound healing [71]. Yu et al. [72] showed that SW therapy attenuated H9c2 myoblast apoptosis by activating the AKT signaling pathway. Zhang et al. [73] demonstrated that LESW downgraded acute myocardial infarction in rats by suppressing the mitochondrial apoptotic pathway. Taken together, LESW therapy has the potential to counteract inflammation, inhibit apoptosis, and promote tissue regeneration in injured tissue.

Zimmermann et al. [74] reported that LESW significantly improved pelvic pain and micturition, as well as erectile function and quality of life, compared with the control group. Yuan et al. [75] performed a systemic review of 6 publications involving 5 randomized controlled trials with 280 patients. They concluded that LESW achieved great efficacy for the treatment of chronic prostatitis/chronic pelvic pain syndrome at the endpoint and during follow-up for 4 and 12 weeks. There were no significant side effects in these studies.

Therefore, we hypothesized that LESW may generate some growth factors or cytokines, and that inhibiting inflammatory reactions and apoptosis might also have therapeutic effects on patients with IC/BPS. Recently, Chuang et al. [76] reported a prospective, randomized, double-blind, placebo-controlled study of extracorporeal SW therapy for IC/BPS. They found no difference in the mean change in IC/BPS symptoms between the LESW and placebo groups. However, a significantly higher proportion of patients who received LESW responded, as shown by an improvement in the visual analogue scale (VAS) of $\geq 3$ points versus placebo $(\mathrm{P}=0.035)$. At 12 weeks posttreatment, $57.1 \%$ of patients in the LESW group showed improvement in the VAS of $\geq 3$ points, versus $19.0 \%$ in the placebo group $(\mathrm{P}=0.011)$. This finding was associated with an improve- ment in frequency (LESW group: $-1.0 \pm 2.3$ vs. placebo: $0.7 \pm$ 3.2; $\mathrm{P}=0.065)$. No significant adverse events were found in either group. We suggest that LESW might reduce bladder pain in IC/BPS patients. More advanced basic science research and clinical trials are warranted before LESW can be widely used for the treatment of IC/BPS.

\section{FUTURE DIRECTIONS}

The experiences obtained from stem cell, PRP, and LESW studies have created new opportunities for the treatment of many incurable diseases. However, while previous publications have reported positive effects in preclinical studies and clinical reports, there are several critical questions to be answered before these methods can be fully incorporated into clinical practice. First, upon searching www.clinicaltrials.gov using key words such as "stem cells," "PRP," "shock wave," "interstitial cystitis," "bladder pain syndrome," and so forth, no major clinical trials are recruiting or underway to investigate the therapeutic effects of these treatments in patients with IC/BPS. As such, multicenter, long-term, and randomized clinical trials are urgently needed to elucidate the efficiency, stability and safety of these regenerative medicine therapies. Second, there are no universal markers or pathological findings to prove the mechanism of action of stem cells, PRP, and LESW. As such, future efforts are necessary in human clinical trials. Finally, although a number of animal models have been established that help to better understand the underlying pathophysiological mechanisms, no animal model can be expected to reproduce all of the various symptoms experienced by human patients [77]. As such, the finding of animal studies cannot fully apply to IC/BPS patients.

\section{AUTHOR CONTRIBUTION STATEMENT}

- Conceptualization: YCC

- Data curation: $Y C H, W C L$

-Writing-original draft: $C C L, Y C H, W C L$

-Writing-review \& editing: $C C L, Y C C$

\section{REFERENCES}

1. Abrams P, Cardozo L, Fall M, Griffiths D, Rosier P, Ulmsten U, et al. The standardisation of terminology of lower urinary tract function: report from the Standardisation Sub-committee of the International Continence Society. Neurourol Urodyn 2002;21:167-78. 
2. Jhang JF, Kuo HC. Pathomechanism of interstitial cystitis/bladder pain syndrome and mapping the heterogeneity of disease. Int Neurourol J 2016;20:S95-104.

3. Berry SH, Elliott MN, Suttorp M, Bogart LM, Stoto MA, Eggers P, et al. Prevalence of symptoms of bladder pain syndrome/interstitial cystitis among adult females in the United States. J Urol 2011; 186:540-4.

4. Suskind AM, Berry SH, Ewing BA, Elliott MN, Suttorp MJ, Clemens JQ. The prevalence and overlap of interstitial cystitis/bladder pain syndrome and chronic prostatitis/chronic pelvic pain syndrome in men: results of the RAND Interstitial Cystitis Epidemiology male study. J Urol 2013;189:141-5.

5. Leppilahti M, Tammela TL, Huhtala H, Auvinen A. Prevalence of symptoms related to interstitial cystitis in women: a population based study in Finland. J Urol 2002;168:139-43.

6. Leppilahti M, Tammela TLJ, Huhtala H, Kiilholma P, Leppilahti K, Auvinen A. Interstitial cystitis-like urinary symptoms among patients with Sjögren's syndrome: a population-based study in Finland. Am J Med 2003;115:62-5.

7. Choe JH, Son H, Song YS, Kim JC, Lee JZ, Lee KS. Prevalence of painful bladder syndrome/interstitial cystitis-like symptoms in women: a population-based study in Korea. World J Urol 2011;29: 103-8.

8. Chung SD, Xirasagar S, Lin CC, Ling W, Li HC, Lin HC. Increased risk of ischemic stroke among women with bladder pain syndrome/interstitial cystitis: a cohort study from Taiwan. Neurourol Urodyn 2015;34:44-9.

9. Braunstein R, Shapiro E, Kaye J, Moldwin R. The role of cystoscopy in the diagnosis of Hunner's ulcer disease. J Urol 2008;180:1383-6.

10. van de Merwe JP, Nordling J, Bouchelouche P, Bouchelouche K, Cervigni M, Daha LK, et al. Diagnostic criteria, classification, and nomenclature for painful bladder syndrome/interstitial cystitis: an ESSIC proposal. Eur Urol 2008;53:60-7.

11. Nordling J, Anjum FH, Bade JJ, Bouchelouche K, Bouchelouche P, Cervigni $\mathrm{M}$, et al. Primary evaluation of patients suspected of having interstitial cystitis (IC). Eur Urol 2004;45:662-9.

12. Ottem DP, Teichman JM. What is the value of cystoscopy with hydrodistension for interstitial cystitis? Urology 2005;66:494-9.

13. Homma Y, Akiyama Y, Tomoe H, Furuta A, Ueda T, Maeda D, et al. Clinical guidelines for interstitial cystitis/bladder pain syndrome. Int J Urol 2020 Apr 14 [Epub]. https://doi.org/10.1111/iju.14234.

14. Birder L, Andersson KE. Urothelial signaling. Physiol Rev 2013;93: 653-80.

15. Parsons CL. The role of a leaky epithelium and potassium in the generation of bladder symptoms in interstitial cystitis/overactive bladder, urethral syndrome, prostatitis and gynaecological chronic pelvic pain. BJU Int 2011;107:370-5.

16. Hauser PJ, Dozmorov MG, Bane BL, Slobodov G, Culkin DJ, Hurst RE. Abnormal expression of differentiation related proteins and proteoglycan core proteins in the urothelium of patients with interstitial cystitis. J Urol 2008;179:764-9.

17. Zeng Y, Wu XX, Homma Y, Yoshimura N, Iwaki H, Kageyama S, et al. Uroplakin III-delta4 messenger RNA as a promising marker to identify nonulcerative interstitial cystitis. J Urol 2007;178:1322-7; discussion 7.

18. Southgate J, Varley CL, Garthwaite MA, Hinley J, Marsh F, Stahlschmidt J, et al. Differentiation potential of urothelium from patients with benign bladder dysfunction. BJU Int 2007;99:150616.

19. Shie JH, Liu HT, Kuo HC. Increased cell apoptosis of urothelium mediated by inflammation in interstitial cystitis/painful bladder syndrome. Urology 2012;79:484.e7-13.

20. Kim J, Keay SK, Freeman MR. Heparin-binding epidermal growth factor-like growth factor functionally antagonizes interstitial cystitis antiproliferative factor via mitogen-activated protein kinase pathway activation. BJU Int 2009;103:541-6.

21. Peeker R, Enebrack L, Fall M, Aldenborg F. Recruitment, distribution and phenotypes of mast cells in interstitial cystitis. J Urol 2000;163:1009-15.

22. Parsons CL, Greene RA, Chung M, Stanford EJ, Singh G. Abnormal urinary potassium metabolism in patients with interstitial cystitis. J Urol 2005;173:1182-5.

23. Gonzalez RR, Fong T, Belmar N, Saban M, Felsen D, Te A. Modulating bladder neuro-inflammation: RDP58, a novel anti-inflammatory peptide, decreases inflammation and nerve growth factor production in experimental cystitis. J Urol 2005;173:630-4.

24. Sant GR, Kempuraj D, Marchand JE, Theoharides TC. The mast cell in interstitial cystitis: role in pathophysiology and pathogenesis. Urology 2007;69:34-40.

25. Warren JW, van de Merwe JP, Nickel JC. Interstitial cystitis/bladder pain syndrome and nonbladder syndromes: facts and hypotheses. Urology 2011;78:727-32.

26. Khullar V, Digesu GA, Veit-Rubin N, Sahai A, Rahnama’i MS, Tarcan $\mathrm{T}$, et al. How can we improve the diagnosis and management of bladder pain syndrome? Part 2:ICI-RS 2018. Neurourol Urodyn 2019;38 Suppl 5:S71-81.

27. Becker C, Jakse G. Stem cells for regeneration of urological structures. Eur Urol 2007;51:1217-28.

28. Kim A, Shin DM, Choo MS. Stem cell therapy for interstitial cystitis/bladder pain syndrome. Curr Urol Rep 2016;17:1. 
29. Sun DZ, Abelson B, Babbar P, Damaser MS. Harnessing the mesenchymal stem cell secretome for regenerative urology. Nat Rev Urol 2019;16:363-75.

30. Song M, Heo J, Chun JY, Bae HS, Kang JW, Kang H, et al. The paracrine effects of mesenchymal stem cells stimulate the regeneration capacity of endogenous stem cells in the repair of a bladderoutlet-obstruction-induced overactive bladder. Stem Cells Dev 2014;23:654-63.

31. Lin CS, Lin G, Lue TF. Allogeneic and xenogeneic transplantation of adipose-derived stem cells in immunocompetent recipients without immunosuppressants. Stem Cells Dev 2012;21:2770-8.

32. Kim JH, Lee HJ, Song YS. Treatment of bladder dysfunction using stem cell or tissue engineering technique. Korean J Urol 2014;55: 228-38.

33. Thomson JA, Itskovitz-Eldor J, Shapiro SS, Waknitz MA, Swiergiel JJ, Marshall VS, et al. Embryonic stem cell lines derived from human blastocysts. Science 1998;282:1145-7.

34. Chhabra A. Derivation of human induced pluripotent stem cell (iPSC) lines and mechanism of pluripotency: historical perspective and recent advances. Stem Cell Rev Rep 2017;13:757-73.

35. Kaiser J, Vogel G. Embryonic stem cells. Controversial ruling throws U.S. research into a tailspin. Science 2010;329:1132-3.

36. Takahashi K, Yamanaka S. Induction of pluripotent stem cells from mouse embryonic and adult fibroblast cultures by defined factors. Cell 2006;126:663-76.

37. Takahashi K, Tanabe K, Ohnuki M, Narita M, Ichisaka T, Tomoda $\mathrm{K}$, et al. Induction of pluripotent stem cells from adult human fibroblasts by defined factors. Cell 2007;131:861-72.

38. Reed-Maldonado AB, Lue TF. The current status of stem-cell therapy in erectile dysfunction: a review. World J Mens Health 2016; 34:155-64.

39. Song M, Lim J, Yu HY, Park J, Chun JY, Jeong J, et al. Mesenchymal stem cell therapy alleviates interstitial cystitis by activating Wnt signaling pathway. Stem Cells Dev 2015;24:1648-57.

40. Xie J, Liu B, Chen J, Xu Y, Zhan H, Yang F, et al. Umbilical cord-derived mesenchymal stem cells alleviated inflammation and inhibited apoptosis in interstitial cystitis via AKT/mTOR signaling pathway. Biochem Biophys Res Commun 2018;495:546-52.

41. Hirose Y, Yamamoto T, Nakashima M, Funahashi Y, Matsukawa Y, Yamaguchi $\mathrm{M}$, et al. Injection of dental pulp stem cells promotes healing of damaged bladder tissue in a rat model of chemically induced cystitis. Cell Transplant 2016;25:425-36.

42. Furuta A, Yamamoto T, Igarashi T, Suzuki Y, Egawa S, Yoshimura $\mathrm{N}$. Bladder wall injection of mesenchymal stem cells ameliorates bladder inflammation, overactivity, and nociception in a chemical- ly induced interstitial cystitis-like rat model. Int Urogynecol J 2018;29:1615-22.

43. Li J, Luo H, Dong X, Liu Q, Wu C, Zhang T, et al. Therapeutic effect of urine-derived stem cells for protamine/lipopolysaccharideinduced interstitial cystitis in a rat model. Stem Cell Res Ther 2017;8:107.

44. Kim A, Yu HY, Lim J, Ryu CM, Kim YH, Heo J, et al. Improved efficacy and in vivo cellular properties of human embryonic stem cell derivative in a preclinical model of bladder pain syndrome. Sci Rep 2017;7:8872.

45. Ryu CM, Yu HY, Lee HY, Shin JH, Lee S, Ju H, et al. Longitudinal intravital imaging of transplanted mesenchymal stem cells elucidates their functional integration and therapeutic potency in an animal model of interstitial cystitis/bladder pain syndrome. Theranostics 2018;8:5610-24.

46. Adamowicz J, Pokrywczynska M, Drewa T. Conditioned medium derived from mesenchymal stem cells culture as a intravesical therapy for cystitis interstitials. Med Hypotheses 2014;82:670-3.

47. Lander EB, Berman MH, See JR. Personal cell therapy for interstitial cystitis with autologous stromal vascular fraction stem cells. Ther Adv Urol 2019;11:1756287219868590.

48. Alves R, Grimalt R. A review of platelet-rich plasma: history, biology, mechanism of action, and classification. Skin Appendage Disord 2018;4:18-24.

49. Magalon J, Chateau AL, Bertrand B, Louis ML, Silvestre A, Giraudo L, et al. DEPA classification: a proposal for standardising PRP use and a retrospective application of available devices. BMJ Open Sport Exerc Med 2016;2:e000060.

50. Ke QS, Jhang JF, Lin TY, Ho HC, Jiang YH, Hsu YH, et al. Therapeutic potential of intravesical injections of platelet-rich plasma in the treatment of lower urinary tract disorders due to regenerative deficiency. Ci Ji Yi Xue Za Zhi 2019;31:135-43.

51. Kwapisz A, Prabhakar S, Compagnoni R, Sibilska A, Randelli P. Platelet-rich plasma for elbow pathologies: a descriptive review of current literature. Curr Rev Musculoskelet Med 2018;11:598-606.

52. Donmez MI, Inci K, Zeybek ND, Dogan HS, Ergen A. The early histological effects of intravesical instillation of platelet-rich plasma in cystitis models. Int Neurourol J 2016;20:188-96.

53. Jhang JF, Wu SY, Lin TY, Kuo HC. Repeated intravesical injections of platelet-rich plasma are effective in the treatment of interstitial cystitis: a case control pilot study. Low Urin Tract Symptoms 2019; 11:042-7.

54. Jhang JF, Lin TY, Kuo HC. Intravesical injections of platelet-rich plasma is effective and safe in treatment of interstitial cystitis refractory to conventional treatment-A prospective clinical trial. Neurou- 
rol Urodyn 2019;38:703-9.

55. Wang CJ. An overview of shock wave therapy in musculoskeletal disorders. Chang Gung Med J 2003;26:220-32.

56. d'Agostino MC, Craig K, Tibalt E, Respizzi S. Shock wave as biological therapeutic tool: from mechanical stimulation to recovery and healing, through mechanotransduction. Int J Surg 2015;24: 147-53.

57. Wang CJ. Extracorporeal shockwave therapy in musculoskeletal disorders. J Orthop Surg Res 2012;7:11.

58. Speed C. A systematic review of shockwave therapies in soft tissue conditions: focusing on the evidence. Br J Sports Med 2014;48: 1538-42.

59. Speed CA. Extracorporeal shock-wave therapy in the management of chronic soft-tissue conditions. J Bone Joint Surg Br 2004;86:16571.

60. Lohrer H, Nauck T, Korakakis V, Malliaropoulos N. Historical ESWT paradigms are overcome: a narrative review. Biomed Res Int 2016;2016:3850461.

61. Werdin F, Tenenhaus M, Rennekampff HO. Chronic wound care. Lancet 2008;372:1860-2.

62. Chancellor MB, Yoshimura N. Treatment of interstitial cystitis. Urology 2004;63:85-92.

63. Lee JD, Lee MH. Increased expression of hypoxia-inducible factorlalpha and vascular endothelial growth factor associated with glomerulation formation in patients with interstitial cystitis. Urology 2011;78:971.e11-5.

64. Chaussy C, Schmiedt E, Jocham D, Brendel W, Forssmann B, Walther V. First clinical experience with extracorporeally induced destruction of kidney stones by shock waves. J Urol 1982;127:417-20.

65. Wang CJ. An overview of shock wave therapy in musculoskeletal disorders. Chang Gung Med J 2003;26:220-32.

66. Di Meglio F, Nurzynska D, Castaldo C, Miraglia R, Romano V, De Angelis A, et al. Cardiac shock wave therapy: assessment of safety and new insights into mechanisms of tissue regeneration. J Cell Mol Med 2012;16:936-42.

67. Vardi Y, Appel B, Kilchevsky A, Gruenwald I. Does low intensity extracorporeal shock wave therapy have a physiological effect on erectile function? Short-term results of a randomized, doubleblind, sham controlled study. J Urol 2012;187:1769-75.
68. Mariotto S, de Prati AC, Cavalieri E, Amelio E, Marlinghaus E, Suzuki H. Extracorporeal shock wave therapy in inflammatory diseases: molecular mechanism that triggers anti-inflammatory action. Curr Med Chem 2009;16:2366-72.

69. Wang HJ, Lee WC, Tyagi P, Huang CC, Chuang YC. Effects of low energy shock wave therapy on inflammatory moleculars, bladder pain, and bladder function in a rat cystitis model. Neurourol Urodyn 2017;36:1440-7.

70. Chen YT, Yang CC, Sun CK, Chiang HJ, Chen YL, Sung PH, et al. Extracorporeal shock wave therapy ameliorates cyclophosphamide-induced rat acute interstitial cystitis though inhibiting inflammation and oxidative stress-in vitro and in vivo experiment studies. Am J Transl Res 2014;6:631-48.

71. Weihs AM, Fuchs C, Teuschl AH, Hartinger J, Slezak P, Mittermayr $\mathrm{R}$, et al. Shock wave treatment enhances cell proliferation and improves wound healing by ATP release-coupled extracellular signalregulated kinase (ERK) activation. J Biol Chem 2014;289:27090104.

72. Yu W, Shen T, Liu B, Wang S, Li J, Dai D, et al. Cardiac shock wave therapy attenuates $\mathrm{H} 9 \mathrm{c} 2$ myoblast apoptosis by activating the AKT signal pathway. Cell Physiol Biochem 2014;33:1293-303.

73. Zhang Y, Shen T, Liu B, Dai D, Cai J, Zhao C, et al. Cardiac shock wave therapy attenuates cardiomyocyte apoptosis after acute myocardial infarction in rats. Cell Physiol Biochem 2018;49:1734-46.

74. Zimmermann R, Cumpanas A, Miclea F, Janetschek G. Extracorporeal shock wave therapy for the treatment of chronic pelvic pain syndrome in males: a randomised, double-blind, placebo-controlled study. Eur Urol 2009;56:418-24.

75. Yuan P, Ma D, Zhang Y, Gao X, Liu Z, Li R, et al. Efficacy of low-intensity extracorporeal shock wave therapy for the treatment of chronic prostatitis/chronic pelvic pain syndrome: a systematic review and meta-analysis. Neurourol Urodyn 2019;38:1457-66.

76. Chuang YC, Meng E, Chancellor M, Kuo HC. Pain reduction realized with extracorporeal shock wave therapy for the treatment of symptoms associated with interstitial cystitis/bladder pain syndrome-A prospective, multicenter, randomized, double-blind, placebo-controlled study. Neurourol Urodyn 2020;39:1505-14.

77. Westropp JL, Buffington CA. In vivo models of interstitial cystitis. J Urol 2002;167:694-702. 